\title{
Further applications of a power series method for pattern avoidance
}

\author{
Narad Rampersad* \\ Department of Mathematics and Statistics \\ University of Winnipeg \\ 515 Portage Avenue \\ Winnipeg, Manitoba R3B 2E9 (Canada) \\ n.rampersad@uwinnipeg.ca
}

Submitted: Jul 31, 2009; Accepted: Jun 10, 2011; Published: Jun 21, 2011

Mathematics Subject Classification: 68R15

\begin{abstract}
In combinatorics on words, a word $w$ over an alphabet $\Sigma$ is said to avoid a pattern $p$ over an alphabet $\Delta$ if there is no factor $x$ of $w$ and no non-erasing morphism $h$ from $\Delta^{*}$ to $\Sigma^{*}$ such that $h(p)=x$. Bell and Goh have recently applied an algebraic technique due to Golod to show that for a certain wide class of patterns $p$ there are exponentially many words of length $n$ over a 4-letter alphabet that avoid $p$. We consider some further consequences of their work. In particular, we show that any pattern with $k$ variables of length at least $4^{k}$ is avoidable on the binary alphabet. This improves an earlier bound due to Cassaigne and Roth.
\end{abstract}

\section{Introduction}

In combinatorics on words, the notion of an avoidable/unavoidable pattern was first introduced (independently) by Bean, Ehrenfeucht, and McNulty [1] and Zimin [22]. Let $\Sigma$ and $\Delta$ be alphabets: the alphabet $\Delta$ is the pattern alphabet and its elements are variables. A pattern $p$ is a non-empty word over $\Delta$. A word $w$ over $\Sigma$ is an instance of $p$ if there exists a non-erasing morphism $h: \Delta^{*} \rightarrow \Sigma^{*}$ such that $h(p)=w$. A pattern $p$ is avoidable if there exists infinitely many words $x$ over a finite alphabet such that no factor of $x$ is an instance of $p$. Otherwise, $p$ is unavoidable. If $p$ is avoided by infinitely many words on an $m$-letter alphabet then it is said to be $m$-avoidable. The survey chapter in Lothaire [12, Chapter 3] gives a good overview of the main results concerning avoidable patterns.

\footnotetext{
*The author is supported by an NSERC Postdoctoral Fellowship.
} 
The classical results of Thue $[19,20]$ established that the pattern $x x$ is 3 -avoidable and the pattern $x x x$ is 2-avoidable. Schmidt [17] (see also [14]) proved that any binary pattern of length at least 13 is 2-avoidable; Roth [15] showed that the bound of 13 can be replaced by 6. Cassaigne [7] and Vaniček [21] (see [10]) determined exactly the set of binary patterns that are 2-avoidable.

Bean, Ehrenfeucht, and McNulty [1] and Zimin [22] characterized the avoidable patterns in general. Let us call a pattern $p$ for which all variables occurring in $p$ occur at least twice a doubled pattern. A consequence of the characterization of the avoidable patterns is that any doubled pattern is avoidable. Bell and Goh [3] proved the much stronger result that every doubled pattern is 4-avoidable. Cassaigne and Roth (see [8] or [12, Chapter 3]) proved that any pattern containing $k$ distinct variables and having length greater than $200 \cdot 5^{k}$ is 2-avoidable. In this note we apply the arguments of Bell and Goh to show the following result, which improves that of Cassaigne and Roth.

Theorem 1. Let $k$ be a positive integer and let $p$ be a pattern containing $k$ distinct variables.

(a) If $p$ has length at least $2^{k}$ then $p$ is 4-avoidable.

(b) If $p$ has length at least $3^{k}$ then $p$ is 3-avoidable.

(c) If $p$ has length at least $4^{k}$ then $p$ is 2-avoidable.

\section{A power series approach}

Rather than simply wishing to show the avoidability of a pattern $p$, one may wish instead to determine the number of words of length $n$ over an $m$-letter alphabet that avoid $p$ (see, for instance, Berstel's survey [4]). Brinkhuis [6] and Brandenburg [5] showed that there are exponentially many words of length $n$ over a 3-letter alphabet that avoid the pattern $x x$. Similarly, Brandenburg showed that there are exponentially many words of length $n$ over a 2-letter alphabet that avoid the pattern $x x x$.

As previously mentioned, Bell and Goh proved that every doubled pattern is 4avoidable. In fact, they proved the stronger result that there are exponentially many words of length $n$ over a 4-letter alphabet that avoid a given doubled pattern. Their main tool in obtaining this result is the following (here $\left[x^{n}\right] G(x)$ denotes the coefficient of $x^{n}$ in the series expansion of $G(x))$.

Theorem 2 (Golod). Let $S$ be a set of words over an m-letter alphabet, each word of length at least 2 . Suppose that for each $i \geq 2$, the set $S$ contains at most $c_{i}$ words of length $i$. If the power series expansion of

$$
G(x):=\left(1-m x+\sum_{i \geq 2} c_{i} x^{i}\right)^{-1}
$$

has non-negative coefficients, then there are least $\left[x^{n}\right] G(x)$ words of length $n$ over an m-letter alphabet that avoid $S$. 
Theorem 2 is a special case of a result originally presented by Golod (see Rowen [16, Lemma 6.2.7]) in an algebraic setting. We have stated it here using combinatorial terminology. The proof given in Rowen's book also is phrased in algebraic terminology; in order to make the technique perhaps a little more accessible to combinatorialists, we present a proof of Theorem 2 using combinatorial language.

Proof of Theorem 2. For two power series $f(x)=\sum_{i \geq 0} a_{i} x^{i}$ and $g(x)=\sum_{i \geq 0} b_{i} x^{i}$, we write $f \geq g$ to mean that $a_{i} \geq b_{i}$ for all $i \geq 0$. Let $F(x):=\sum_{i \geq 0} a_{i} x^{i}$, where $a_{i}$ is the number of words of length $i$ over an $m$-letter alphabet that avoid $S$. Let $G(x):=\sum_{i \geq 0} b_{i} x^{i}$ be the power series expansion of $G$ defined above. We wish to show $F \geq G$.

For $k \geq 1$, there are $m^{k}-a_{k}$ words $w$ of length $k$ over an $m$-letter alphabet that contain a word in $S$ as a factor. On the other hand, for any such $w$ either (a) $w=w^{\prime} a$, where $a$ is a single letter and $w^{\prime}$ is a word of length $k-1$ containing a word in $S$ as a factor; or (b) $w=x y$, where $x$ is a word of length $k-j$ that avoids $S$ and $y \in S$ is a word of length $j$. There are at most $\left(m^{k-1}-a_{k-1}\right) m$ words $w$ of the form (a), and there are at most $\sum_{j} a_{k-j} c_{j}$ words $w$ of the form (b). We thus have the inequality

$$
m^{k}-a_{k} \leq\left(m^{k-1}-a_{k-1}\right) m+\sum_{j} a_{k-j} c_{j}
$$

Rearranging, we have

$$
a_{k}-a_{k-1} m+\sum_{j} a_{k-j} c_{j} \geq 0
$$

for $k \geq 1$.

Consider the function

$$
\begin{aligned}
H(x) & :=F(x)\left(1-m x+\sum_{j \geq 2} c_{j} x^{j}\right) \\
& =\left(\sum_{i \geq 0} a_{i} x^{i}\right)\left(1-m x+\sum_{j \geq 2} c_{j} x^{j}\right) .
\end{aligned}
$$

Observe that for $k \geq 1$, we have $\left[x^{k}\right] H(x)=a_{k}-a_{k-1} m+\sum_{j} a_{k-j} c_{j}$. By (2), we have $\left[x^{k}\right] H(x) \geq 0$ for $k \geq 1$. Since $\left[x^{0}\right] H(x)=1$, the inequality $H \geq 1$ holds, and in particular, $H-1$ has non-negative coefficients. We conclude that $F=H G=(H-1) G+G \geq G$, as required.

Theorem 2 bears a certain resemblance to the Goulden-Jackson cluster method [11, Section 2.8], which also produces a formula similar to (1). The cluster method yields an exact enumeration of the words avoiding the set $S$ but requires $S$ to be finite. By contrast, Theorem 2 only gives a lower bound on the number of words avoiding $S$, but now the set $S$ can be infinite.

Theorem 2 can be viewed as a non-constructive method to show the avoidability of patterns over an alphabet of a certain size. In this sense it is somewhat reminiscent of 
the probabilistic approach to pattern avoidance using the Lovász local lemma (see [2, 9]). For pattern avoidance it may even be more powerful than the local lemma in certain respects. For instance, Pegden [13] proved that doubled patterns are 22-avoidable using the local lemma, whereas Bell and Goh were able to show 4-avoidability using Theorem 2. Similarly, the reader may find it a pleasant exercise to show using Theorem 2 that there are infinitely many words avoiding $x x$ over a 7-letter alphabet; as far as we are aware, the smallest alphabet size for which the avoidability of $x x$ has been shown using the local lemma is 13 [18].

\section{Proof of Theorem 1}

To prove Theorem 1 we begin with some lemmas.

Lemma 3. Let $k \geq 1$ and $m \geq 2$ be integers. If $w$ is a word of length at least $m^{k}$ over a $k$-letter alphabet, then $w$ contains a non-empty factor $w^{\prime}$ such that the number of occurrences of each letter in $w^{\prime}$ is a multiple of $m$.

Proof. Suppose $w$ is over the alphabet $\Sigma=\{1,2, \ldots, k\}$. Define the map $\psi: \Sigma^{*} \rightarrow \mathbb{N}^{k}$ that maps a word $x$ to the $k$-tuple $\left[|x|_{1} \bmod m, \ldots,|x|_{k} \bmod m\right]$, where $|x|_{a}$ denotes the number of occurrences of the letter $a$ in $x$. For each prefix $w_{i}$ of length $i$ of $w$, let $v_{i}=\psi\left(w_{i}\right)$. Since $w$ has length at least $m^{k}, w$ has at least $m^{k}+1$ prefixes, but there are at most $m^{k}$ distinct tuples $v_{i}$. There exists therefore $i<j$ such that $v_{i}=v_{j}$. However, if $w^{\prime}$ is the suffix of $w_{j}$ of length $j-i$, then $\psi\left(w^{\prime}\right)=v_{j}-v_{i}=[0, \ldots, 0]$, and hence the number of occurrences of each letter in $w^{\prime}$ is a multiple of $m$.

Lemma 4 ([3]). Let $k \geq 1$ be an integer and let $p$ be a pattern over the pattern alphabet $\left\{x_{1}, \ldots, x_{k}\right\}$. Suppose that for $1 \leq i \leq k$, the variable $x_{i}$ occurs $a_{i} \geq 1$ times in $p$. Let $m \geq 2$ be an integer and let $\Sigma$ be an m-letter alphabet. Then for $n \geq 1$, the number of words of length $n$ over $\Sigma$ that are instances of the pattern $p$ is at most $\left[x^{n}\right] C(x)$, where

$$
C(x):=\sum_{i_{1} \geq 1} \cdots \sum_{i_{k} \geq 1} m^{i_{1}+\cdots+i_{k}} x^{a_{1} i_{1}+\cdots+a_{k} i_{k}} .
$$

For the proof of the next result, we essentially follow the approach of Bell and Goh.

Theorem 5. Let $k \geq 2$ be an integer and let $p$ be a pattern over a $k$-letter pattern alphabet such that every variable occurring in $p$ occurs at least $\mu$ times.

(a) If $\mu=3$, then for $n \geq 0$, there are at least $2.94^{n}$ words of length $n$ avoiding $p$ over a 3-letter alphabet.

(b) If $\mu=4$, then for $n \geq 0$, there are at least $1.94^{n}$ words of length $n$ avoiding $p$ over a 2-letter alphabet. 
Proof. Let $(m, \mu) \in\{(3,3),(2,4)\}$ and let $\Sigma$ be an $m$-letter alphabet. Define $S$ to be the set of all words over $\Sigma$ that are instances of the pattern $p$. By Lemma 4, the number of words of length $n$ in $S$ is at most $\left[x^{n}\right] C(x)$, where

$$
C(x):=\sum_{i_{1} \geq 1} \cdots \sum_{i_{k} \geq 1} m^{i_{1}+\cdots+i_{k}} x^{a_{1} i_{1}+\cdots+a_{k} i_{k}}
$$

and for $1 \leq i \leq k$ we have $a_{i} \geq \mu$. Define

$$
B(x):=\sum_{i \geq 0} b_{i} x^{i}=(1-m x+C(x))^{-1},
$$

and set $\lambda:=m-0.06$ (this is not necessarily the optimal value for $\lambda$ ). We claim that $b_{n} \geq \lambda b_{n-1}$ for all $n \geq 0$. This suffices to prove the lemma, as we would then have $b_{n} \geq \lambda^{n}$ and the result follows by an application of Theorem 2 .

We prove the claim by induction on $n$. When $n=0$, we have $b_{0}=1$ and $b_{1}=m$. Since $m>\lambda$, the inequality $b_{1} \geq \lambda b_{0}$ holds, as required. Suppose that for all $j<n$, we have $b_{j} \geq \lambda b_{j-1}$. Since $B=(1-m x+C)^{-1}$, we have $B(1-m x+C)=1$. Hence $\left[x^{n}\right] B(1-m x+C)=0$ for $n \geq 1$. However,

$$
B(1-m x+C)=\left(\sum_{i \geq 0} b_{i} x^{i}\right)\left(1-m x+\sum_{i_{1} \geq 1} \cdots \sum_{i_{k} \geq 1} m^{i_{1}+\cdots+i_{k}} x^{a_{1} i_{1}+\cdots+a_{k} i_{k}}\right),
$$

SO

$$
\left[x^{n}\right] B(1-m x+C)=b_{n}-b_{n-1} m+\sum_{i_{1} \geq 1} \cdots \sum_{i_{k} \geq 1} m^{i_{1}+\cdots+i_{k}} b_{n-\left(a_{1} i_{1}+\cdots+a_{k} i_{k}\right)}=0 .
$$

Rearranging, we obtain

$$
b_{n}=\lambda b_{n-1}+(m-\lambda) b_{n-1}-\sum_{i_{1} \geq 1} \cdots \sum_{i_{k} \geq 1} m^{i_{1}+\cdots+i_{k}} b_{n-\left(a_{1} i_{1}+\cdots+a_{k} i_{k}\right)} .
$$

To show $b_{n} \geq \lambda b_{n-1}$ it therefore suffices to show

$$
(m-\lambda) b_{n-1}-\sum_{i_{1} \geq 1} \cdots \sum_{i_{k} \geq 1} m^{i_{1}+\cdots+i_{k}} b_{n-\left(a_{1} i_{1}+\cdots+a_{k} i_{k}\right)} \geq 0 .
$$


Since $b_{j} \geq \lambda b_{j-1}$ for all $j<n$, we have $b_{n-i} \leq b_{n-1} / \lambda^{i-1}$ for $1 \leq i \leq n$. Hence

$$
\begin{aligned}
\sum_{i_{1} \geq 1} \cdots \sum_{i_{k} \geq 1} m^{i_{1}+\cdots+i_{k}} b_{n-\left(a_{1} i_{1}+\cdots+a_{k} i_{k}\right)} & \leq \sum_{i_{1} \geq 1} \cdots \sum_{i_{k} \geq 1} m^{i_{1}+\cdots+i_{k}} \frac{\lambda b_{n-1}}{\lambda^{a_{1} i_{1}+\cdots+a_{k} i_{k}}} \\
& =\lambda b_{n-1} \sum_{i_{1} \geq 1} \cdots \sum_{i_{k} \geq 1} \frac{m^{i_{1}+\cdots+i_{k}}}{\lambda^{a_{1} i_{1}+\cdots+a_{k} i_{k}}} \\
& =\lambda b_{n-1} \sum_{i_{1} \geq 1} \frac{m^{i_{1}}}{\lambda^{a_{1} i_{1}}} \cdots \sum_{i_{k} \geq 1} \frac{m^{i_{k}}}{\lambda^{a_{k} i_{k}}} \\
& \leq \lambda b_{n-1} \sum_{i_{1} \geq 1} \frac{m^{i_{1}}}{\lambda^{\mu i_{1}}} \cdots \sum_{i_{k} \geq 1} \frac{m^{i_{k}}}{\lambda^{\mu i_{k}}} \\
& =\lambda b_{n-1}\left(\sum_{i \geq 1} \frac{m^{i}}{\lambda^{\mu i}}\right)^{k} \\
& =\lambda b_{n-1}\left(\frac{m / \lambda^{\mu}}{1-m / \lambda^{\mu}}\right)^{k} \\
& =\lambda b_{n-1}\left(\frac{m}{\lambda^{\mu}-m}\right)^{k} \\
& \leq \lambda b_{n-1}\left(\frac{m}{\lambda^{\mu}-m}\right)^{2}
\end{aligned}
$$

In order to show that (3) holds, it thus suffices to show that

$$
m-\lambda \geq \lambda\left(\frac{m}{\lambda^{\mu}-m}\right)^{2} .
$$

Recall that $m-\lambda=0.06$. For $(m, \mu)=(3,3)$ we have

$$
2.94\left(\frac{3}{2.94^{3}-3}\right)^{2}=0.052677 \cdots \leq 0.06
$$

and for $(m, \mu)=(2,4)$ we have

$$
1.94\left(\frac{2}{1.94^{4}-2}\right)^{2}=0.052439 \cdots \leq 0.06
$$

as required. This completes the proof of the inductive claim and the proof of the lemma.

We can now complete the proof of Theorem 1. Let $p$ be a pattern with $k$ variables. If $p$ has length at least $2^{k}$, then by Lemma 3 , the pattern $p$ contains a non-empty factor $p^{\prime}$ such that each variable occurring in $p^{\prime}$ occurs at least twice. However, Bell and Goh showed that such a $p^{\prime}$ is 4 -avoidable and hence $p$ is 4 -avoidable. 
Similarly, if $p$ has length at least $3^{k}$ (resp. $4^{k}$ ), then by Lemma 3 , the pattern $p$ contains a non-empty factor $p^{\prime}$ such that each variable occurring in $p^{\prime}$ occurs at least 3 times (resp. 4 times). If $p^{\prime}$ contains only one distinct variable, recall that we have already noted in the introduction that the pattern $x x x$ is 2-avoidable (and hence also 3 -avoidable). If $p^{\prime}$ contains at least two distinct variables, then by Theorem 5 , the pattern $p^{\prime}$ is 3 -avoidable (resp. 2-avoidable), and hence the pattern $p$ is 3 -avoidable (resp. 2-avoidable). This completes the proof of Theorem 1.

Recall that Cassaigne and Roth showed that any pattern $p$ over $k$ variables of length greater than $200 \cdot 5^{k}$ is 2-avoidable. Their proof is constructive but is rather difficult. We are able to obtain the much better bound of $4^{k}$ non-constructively by a somewhat simpler argument. Cassaigne suggests (see the open problem [12, Problem 3.3.2]) that

the bound of $3^{k}$ in Theorem 1(b) can perhaps be replaced by $2^{k}$ and that the bound of $4^{k}$ in Theorem 1(c) can perhaps be replaced by $3 \cdot 2^{k}$. Note that the bound of $2^{k}$ in Theorem 1(a) is optimal, since the Zimin pattern on $k$-variables (see [12, Chapter 3]) has length $2^{k}-1$ and is unavoidable.

\section{Acknowledgments}

We thank Terry Visentin for some helpful discussions concerning Theorem 2 and the Goulden-Jackson cluster method.

\section{References}

[1] D. R. Bean, A. Ehrenfeucht, G. F. McNulty, "Avoidable patterns in strings of symbols", Pacific J. Math. 85 (1979), 261-294.

[2] J. Beck, "An application of Lovász local lemma: there exists an infinite 01-sequence containing no near identical intervals", in Infinite and Finite Sets (A. Hajnal et al. eds.), Colloq. Math. Soc. J. Bolyai 37, 1981, pp. 103-107.

[3] J. Bell, T. L. Goh, "Lower bounds for pattern avoidance", Inform. and Comput. 205 (2007), 1295-1306.

[4] J. Berstel, "Growth of reptition-free words-a review", Theoret. Comput. Sci. 340 (2005), 280-290.

[5] F.-J. Brandenburg, "Uniformly growing $k$-th power-free homomorphisms", Theoret. Comput. Sci. 23 (1983), 69-82.

[6] J. Brinkhuis, "Nonrepetitive sequences on three symbols", Quart. J. Math. Oxford 34 (1983), 145-149.

[7] J. Cassaigne, "Unavoidable binary patterns", Acta Inform. 30 (1993), 385-395.

[8] J. Cassaigne, Motifs évitables et régularités dans les mots, Thèse de doctorat, Université Paris 6, LITP research report TH 94-04. 
[9] J. Currie, "Pattern avoidance: themes and variations", Theoret. Comput. Sci. 339 (2005), 7-18.

[10] P. Goralčik, T. Vaniček, "Binary patterns in binary words", Int. J. Algebra Comput. 1, 387-391.

[11] I. Goulden, D. Jackson, Combinatorial Enumeration, Dover, 2004.

[12] M. Lothaire, Algebraic Combinatorics on Words, Cambridge, 2002.

[13] W. Pegden, "Highly nonrepetitive sequences: winning strategies from the Local Lemma". Manuscript available at http://people.cs.uchicago.edu/ wes/ seqgame.pdf.

[14] N. Rampersad, "Avoiding sufficiently large binary patterns", Bull. Europ. Assoc. Theoret. Comput. Sci. 95 (2008), 241-245.

[15] P. Roth, "Every binary pattern of length six is avoidable on the two-letter alphabet", Acta Inform. 29 (1992), 95-107.

[16] L. Rowen, Ring Theory. Vol. II, Pure and Applied Mathematics 128, Academic Press, Boston, 1988.

[17] U. Schmidt, "Avoidable patterns on two letters", Theoret. Comput. Sci. 63 (1989), $1-17$.

[18] J. Shallit, Unpublished lecture notes.

[19] A. Thue, "Über unendliche Zeichenreihen", Kra. Vidensk. Selsk. Skrifter. I. Mat. Nat. Kl. 7 (1906), 1-22.

[20] A. Thue, "Über die gegenseitige Lage gleicher Teile gewisser Zeichenreihen", Kra. Vidensk. Selsk. Skrifter. I. Math. Nat. Kl. 1 (1912), 1-67.

[21] T. Vaniček, Unavoidable Words, Diploma thesis, Charles University, Prague, 1989.

[22] A. I. Zimin, "Blocking sets of terms", Math. USSR Sbornik 47 (1984), 353-364. 